\title{
Substance Use Route of Administration
}

National Cancer Institute

\section{Source}

National Cancer Institute. Substance Use Route of Administration. NCI Thesaurus. Code C83426.

The method a substance was introduced to a subject. 